\title{
Uso De Espironolactona Em Pacientes Com Insuficiência Cardíaca Em Hospital Público Terciário No Brasil
}

\author{
Spagnol, M.F.; Wajner, A.; Brauner, J.S.; Aguiar, F.M.; Dias, P.S.; Maccarini, \\ J.L.; Waldemar, F.S.; \\ Apresentador: Julia De Luca Maccarini
}

\section{Resumo}

Introdução: A insuficiência cardíaca (IC) é uma doença crônica comum na atualidade. A necessidade de uma intervenção terapêutica adequada é justificada pela sua alta morbidade e mortalidade. O uso de antagonistas da aldosterona, como a espironolactona, mostrou-se eficaz em alguns estudos. Objetivo:

Relacionar o uso de espironolactona e desfechos clínicos, reinternação ou visita à emergência em 30 dias, em pacientes com IC em um hospital terciário do sul do Brasil. Objetivo secundário: Descrição da população estudada avaliação dos óbitos intrahospitalares avaliação das medicações prescritas na última prescrição hospitalar. Materiais e Métodos: Coorte histórica de pacientes internados com diagnóstico de IC pelo escore de Charlson em 2009 e 2010 no Hospital Nossa Senhora da Conceição de Porto Alegre. Foram incluídos apenas os pacientes com Fração de Ejeção (FE) menor que 40\% e idade maior que dezoito anos. Resultados: Apenas 33,7\% da população estudada estava em uso de espironolactona. O Odds ratio para o desfecho combinado foi de Odds Ratio 0,64(0,38-1,08) p=0.09 . Dos pacientes que receberam prescrição de espironolactona $36 \%$ não estavam em uso da terapêutica básica com iECA/BRA e betabloqueador. Conclusão: Não houve associação entre o uso de espironolactona e o desfecho combinado apesar de uma forte tendência de diminuição de risco.

\section{Referência:}

Spagnol, M.F.; Wajner, A.; Brauner, J.S.; Aguiar, F.M.; Dias, P.S.; Maccarini, J.L.; Waldemar, F.S.;. Uso De Espironolactona Em Pacientes Com Insuficiência Cardíaca Em Hospital Público Terciário No Brasil. In: Il Congresso Brasileiro de Medicina Hospitalar - II CBMH [= Blucher Medical Proceedings, vol.1, num.5] São Paulo: Editora Blucher, 2014. p.98 DOI 10.5151/medpro-II-cbmh-098 